\title{
Response to the letter regarding article "Metabolic syndrome is associated with different clinical outcome after cardiac resynchronization therapy in patients with ischemic and non-ischemic cardiomyopathy"
}

We would like to thank the Authors for the commentary to our paper [1]. The commentary furthermore highlighted the importance of fuel metabolism in failing heart and significance of the intervention strategies, which may directly or indirectly target major metabolic pathways balancing glucose and free fatty acids metabolism. It should be noted that in Multicenter Automatic Deffibrillator Implantation Trial with Cardiac Resynchronization Therapy (MADIT-CRT) population, only 15 subjects were treated with trimetazidine, not allowing further evaluation.

Our report demonstrated that patients with metabolic syndrome (MS) depending on etiology for heart failure (HF) presented with different response to cardiac resynchronization therapy (CRT). MS patients derive significant benefit from CRT if present with non-ischemic cardiomyopathy, whereas obese patients without MS show no significant reduction in events. This study suggests that the risk for HF and death may be modulated by presence of MS, a clinical surrogate for insulin resistance [1].

The current hypothesis is that CRT, among other effects, for example on cardiac remodeling, may also improve fuel metabolism of the failing heart. It is possible that improvement in fuel metabolism may correspond to better clinical response to $\mathrm{CRT}$ and reduction in risk for $\mathrm{HF}$ and death. In animal studies, CRT treatment was attributed to activation of important metabolic and insulin signal transduction protein kinase $\mathrm{B}$ pathway $(\mathrm{PKB})$ known as Akt [2]. It is generally accepted that falling heart switches its fuel metabolism from long chain fatty acids to glucose [3] but in people with MS with insulin resistance as predominant pathological factor, glucose is less available for utilization. Therefore, activation of defective PKB/AKt by CRT may potentially modulate glucose uptake in the failing heart.

Although it is only speculative, this study also suggests that intrinsic properties of myocardium fuel metabolism affected by MS may play a role in response to CRT. Future studies are needed to investigate mechanism of CRT in relation to myocardium fuel metabolism and cellular responses in falling heart.

Conflict of interest: None declared

\section{References}

1. Szepietowska B, McNitt S, Polonsky B et al. Metabolic syndrome is associated with different clinical outcome after cardiac resynchronization therapy in patients with ischemic and non-ischemic cardiomyopathy. Cardiol J, 2016; 23: 344-351. doi: 10.5603/ CJ.a2016.0017.

2. Kirk JA, Holewinski RJ, Kooij V et al. Cardiac resynchronization sensitizes the sarcomere to calcium by reactivating GSK-3beta. J Clin Invest, 2014; 124: 129-138.

3. Carley AN, Taegtmeyer H, Lewandowski ED. Matrix revisited: Mechanisms linking energy substrate metabolism to the function of the heart. Circ Res, 2014; 114: 717-729. doi: 10.1161/ CIRCRESAHA.114.301863.

Barbara Szepietowska, Wojciech Zareba University of Rochester Medical Center, 265 Crittenden Blvd., PO Box 653, Rochester, NY 14642, United States, tel: 585-275-5391, fax: 585-273-5283, e-mail: barbara.szepietowska@heart.rochester.edu 